\title{
LA CONSTRUCCIÓN DEL CONOCIMIENTO DESDE EL ENFOQUE INTERCULTURAL
}

\author{
BUILDING KNOWLEDGE FROM AN INTERCULTURAL PERSPECTIVE
}

\author{
Beatriz Malik Liévano* y Belén Ballesteros Velázquez*
}

\begin{abstract}
En este artículo ofrecemos una reflexión sobre nuestra propia trayectoria de Grupo INTER de investigación intercultural, partiendo de lo intercultural como mirada que contempla y permite reconocer la diversidad y, consecuentemente, la complejidad de las situaciones sociales y educativas. Por ello el enfoque intercultural se propone como interacción, comunicación y diálogo, resaltando la arbitrariedad de cualquier clasificación.

Lo intercultural es así una forma de construcción del conocimiento que cuestiona la verdad establecida como forma única de explicación. La complejidad solo puede ser abordada en un diálogo de saberes que restablezca la voz de las personas, evitando la separación entre objeto y sujeto en el avance de la ciencia. El conocimiento científico adquiere sentido en cuanto ofrece una oportunidad de emancipación y empoderamiento de las personas y grupos.

Desde esta perspectiva la práctica de nuestra investigación no está exenta de dilemas, algunos de los cuales se reflejan en este artículo. Problematizamos el uso de categorías a partir de su necesidad y riesgo. Y problematizamos también la idea del relativismo cultural, que siendo herramienta necesaria para la comprensión desde otra lógica posible, plantea tensiones en la investigación aplicada.

Palabras claves: Enfoque intercultural, diversidad, epistemología, complejidad, diálogo de saberes, emancipación.
\end{abstract}

In this article we reflect on our own trajectory within the INTER Group of research in intercultural education, considering that the intercultural approach allows us to regard and acknowledge diversity in a positive way, and consequently, the complexity of social and educational situations. Interculturalism is thus proposed as interaction, communication and dialog, highlighting the arbitrariness of any classification imposed on people.

Interculturalism is a type of knowledge construction that questions the established truth as the only way of explanation. Complexity can only be addressed through a dialogue of different types of knowledge and "know-how" giving voice to people, thus avoiding the artificial divide between object and subject in the advancement of science. Scientific knowledge becomes purposeful because it offers an opportunity of emancipation and empowerment of people and groups. From this perspective, our research is not without dilemmas and challenges, some of which are discussed in this article.

We problematize the use of categories and the risk they entail, in spite of their need. And we also problematize the idea of cultural relativism, which, though a necessary tool in our research from a different logic, raises a few tensions in applied research.

Key word: Intercultural approach, diversity, epistemology, complexity, knowledge dialogue, emancipation.

\section{Introducción: Lo intercultural como paradigma para pensar la diversidad}

Cuando hablamos del enfoque intercultural, o de interculturalidad en general, nos referimos a una forma de posicionarnos en el mundo, de mirar la realidad que nos rodea a través del prisma de la diversidad y la complejidad que nos caracteriza. Esto implica, entre otras cosas, considerar la diversidad como inherente al ser humano y no como un déficit que necesita compensarse. Tal diversidad, lejos de ser una excepción, es la norma en cualquier grupo, por lo que no debería ser posible categorizar a las personas. El enfoque intercultural implica también una toma de postura ética respecto de las relaciones entre las personas.
Hace ya casi dos décadas, Xavier Lluch y Jesús Salinas, de la Asociación de Enseñantes con Gitanos (1996), definían el interculturalismo como una concepción teórica y práctica de carácter universal que atiende la diversidad cultural de todas las sociedades desde los principios de igualdad, interacción y transformación social. Implica una opción ética e ideológica de carácter personal, una forma de entender y vivir las relaciones sociales y una manera de plantear y desarrollar la educación.

Siguiendo a estos autores, podemos destacar cinco elementos que caracterizan este concepto y lo distinguen de otras concepciones de las relaciones entre personas: reconocimiento de la diversidad (opción teórica), defensa de la igualdad (opción ideológica), fomento de la interacción (opción

* Universidad Nacional de Educación a Distancia (UNED), Madrid, España. Correos electrónicos: bmalik@edu.uned.es; bballesteros@edu.uned.es 
ética), dinámica de la transformación social (opción sociopolítica), y promoción de procesos educativos que planteen la interacción cultural en condiciones de equidad (opción educativa).

Atendiendo al significado etimológico del prefijo inter, lo intercultural supone intercambio, interacción e interdependencia, que a su vez conlleva colaboración, construcción conjunta del conocimiento, comunicación, negociación, etc. Hablar de "cultura" implica el reconocimiento de valores, estilos de vida y representaciones simbólicas (cosmovisiones), que los individuos ponen en juego al relacionarse con otras personas y en su comprensión del mundo (Rey, 2010). El término intercultural, por tanto, incluye el abanico de interacciones que ocurren dentro de una "cultura", así como entre "culturas", que están en continuo cambio en sus dimensiones de espacio y tiempo (son dinámicas, no estáticas).

En este sentido, lo intercultural se diferencia del término multicultural, ya que este último no implica interacción, sino una yuxtaposición de grupos diversos, que conviven en espacios cercanos (incluso en el mismo espacio), sin que exista una relación de reciprocidad o de cooperación mutua. Todos los grupos humanos y sociedades son multiculturales (o pluriculturales) por naturaleza, sin embargo, lo intercultural no siempre caracteriza las relaciones en estos contextos.

Como afirman Mata y Ávila (2010:61) "lo intercultural no es un adjetivo para ser aplicado a la escuela, la educación, la gestión, la mediación,... sino una mirada hacia la diversidad, una manera de entenderla y una metáfora para expresarla". Retomando la idea de "las metáforas que nos piensan" de Lizcano (2006), Aguado (2009:13), propone "pensar lo intercultural como metáfora de la diversidad, es decir, como mirada que contempla y permite pensar la diversidad y, por ende, la complejidad de las situaciones sociales y educativas". Esta forma de pensar lo diverso y lo complejo implica distinguir diversidad de diferencia y lo cultural de la cultura.

Como afirma esta autora, habitualmente se establecen diferencias y se "reconocen" grupos culturales a priori. Se confunde diversidad cultural con categorización social. Es muy frecuente en distintos contextos identificar diversidad cultural con origen nacional, lengua materna, nivel económico, etnia, religión, género y otras. El mayor problema es que estas categorías sociales informan sobre grupos sociales en los que los individuos son inscritos o adscritos; pero no nos dan información sobre las personas mismas. Reconocer la diversidad cultural implica reconocer a las personas huyendo de adscripciones previas, fijas y categorizadoras. Esto no es fácil, porque los seres humanos tendemos a pensar en categorías, para poder ordenar y procesar la información que le llega del exterior. Pero es fundamental ser conscientes de esto y no encasillar a las personas (a otros y a nosotros mismos) en categorías preestablecidas, que generan estereotipos, y van asociadas a unas expectativas concretas según el grupo en que se nos adscriba (de capacidad, de "patrones" culturales, de intereses, etc.), que no permiten conocernos realmente, y que nos condicionan en muchas aspectos. En educación esta categorización puede tener consecuencias muy negativas por las expectativas que van asociadas a dichas categorías y los estereotipos que se reproducen.

La diversidad es una constante humana y se define como proceso más que como categoría. La diversidad cultural es un hecho, las heterogeneidades la norma. La (supuesta) homogeneidad es producto de una acción deliberada de ordenar, clasificar, controlar (política, social, psicológica o educativamente). Sin embargo, la mirada que nos permite clasificar, organizar, nombrar las cosas, las experiencias y a las personas no es única ni la misma para todos. La diversidad tiene que ver con el reconocimiento de otras formas de ver el mundo; lo relativo de cualquier clasificación y las consecuencias de la misma. Imponer una clasificación, un nombre, una designación a algo es una forma de ejercer el poder sobre el otro (Aguado, 2009; 2010).

Por otro lado, el enfoque implica reconocer que lo cultural está siempre presente en las experiencias educativas, humanas; y al mismo tiempo reconocer que a priori no sabemos cómo se manifiesta e influye. Esto implica evitar visiones simplistas y sesgadas de la diversidad que la asocian a categorías previamente establecidas, así como asumir que diversidad es complejidad e intersubjetividad (Aguado, 2010; Mata y Ávila, 2010).

Para Abdallah-Pretceille (2006), los peligros más graves derivados de describir a las personas, estudiantes o grupos en función de diferencias es que implican una apreciación moral, surgen inmediatamente valoraciones de mejor o peor, bueno o malo. La consecuencia educativa de tener en cuenta o "respetar las diferencias culturales" es adoptar un modelo de déficit desde el que se 
justifican modelos compensatorios y remediales. Estamos de acuerdo en que al reificar la diferencia, marcamos barreras a veces insalvables, definimos un problema sin solución posible, ya que la única solución sería "dejar de ser la persona que uno es" (Aguado, 2009).

En la fase de análisis de necesidades de un proyecto eurolatinoamericano en el que hemos participado, analizamos la imagen de la diversidad que teníamos en los países participantes. Mata y Ávila (2010), coordinadoras del informe global de este análisis de necesidades, al sintetizar los informes locales, constataron que la diversidad tiende a entenderse como riqueza, asociada de manera abstracta a idearios positivos: aportación, diálogo, pluralidad, mezcla, etc. Sin embargo, cuando tiene que personalizarse no se siente como propia: los diversos siempre son los otros. La cultura tiende a verse como algo estático y, por lo tanto, la diversidad cultural se entiende desde la diferencia entre grupos estáticos (asociados a referencias étnicas) que tienen culturas diferentes y monolíticas, negando la diversidad de cada individuo dentro de dichos grupos. Así por ejemplo, están los inmigrantes, indígenas, los desplazados, los travellers, etc. De esto se concluye que los diversos siempre son los otros, personas que en muchos casos son víctimas de procesos de marginación o racismo y a las que solemos referirnos utilizando cierto paternalismo o falsa tolerancia. Nuestros países, por tanto, no serían diversos sin estos grupos (Mata y Ávila, 2010). En función de lo que hemos defendido anteriormente respecto de la diversidad y el enfoque intercultural, esto sería imposible, ya que todas las personas somos diversas. Esta es una contracción con la que nos encontramos habitualmente, tanto en nuestro propio grupo de investigación, como en el trabajo de otros grupos (nos referimos solo a aquellos grupos o autores que comparten las premisas del enfoque intercultural como lo entendemos nosotras. Hay otros autores/grupos trabajando en temas de interculturalidad para quienes esto no supone una contradicción porque en su concepción del enfoque intercultural, la diversidad sí se define en función de categorías específicas).

Así, reconocemos que todos los contextos son diversos y no consideramos necesario describir a las personas en función de esas categorías a las que nos referíamos antes (nacionalidad, género, etnia, etc.), o de características más visibles. Sin embargo, a la hora de compartir estas ideas con otros interlocutores, o de formular proyectos para presentar a convocatorias oficiales, nos encontramos con que no se comprende esta forma de mirar la diversidad, esta apuesta por reconocer a las personas en toda su complejidad y no encasillar en un grupo determinado. Se interpreta que no estamos "mirando" o investigando en el lugar adecuado si la diversidad no es "visible" en ese contexto (según categorías predeterminadas), e incluso se nos penaliza por no definir grupos a priori. En este sentido, las políticas educativas y los organismos que financian proyectos de investigación condicionan de manera muy significativa la investigación que se lleva a cabo en nuestro país (en España, pero probablemente esté ocurriendo en otros países), y están perjudicando de forma alarmante los ideales de equidad y justicia social y concretamente la consecución de objetivos que deberían ser prioritarios en educación, tan básicos como que se cumpla el derecho de todos los niños y jóvenes (y adultos en los niveles que corresponda) a recibir una educación de calidad con independencia de etiquetas y categorías establecidas a priori que de forma sutil, pero con resultados negativos visibles, discriminan y afectan a muchas personas.

En el ámbito escolar, la educación intercultural debe dirigirse a todo el alumnado, a todos los centros, e implica garantizar que los objetivos educativos que nos planteamos como valiosos sean alcanzados por todas y todos y que pueden ayudarnos a desenvolvernos de manera más fácil en los distintos contextos en los que nos movemos (Téllez, Mata, Sutil y Malik, 2005), valorando la diversidad presente en cualquier aula y contexto.

No obstante, frecuentemente la imagen de la diversidad ofrecida por las diferentes legislaciones y normativas educativas lleva implícita, e incluso explícita en ocasiones, la idea de déficit o problema, así como un tratamiento específico o compensatorio. Se constata, por otra parte, que la distancia entre declaraciones y prácticas, pese al avance registrado en términos de desarrollo legislativo, es aún muy grande. La necesidad de cambio y de transformación profunda de las estructuras educativas resulta evidente, ya que las actuales no permiten el reconocimiento de la diversidad. Un factor importante de esa transformación está en los docentes, por lo que la formación del profesorado en el enfoque intercultural se señala como prioridad en varios informes (Mata y Ávila, 2010). Podemos concluir con estas autoras, que a pesar de que la educación intercultural ha sido una 
línea prioritaria en las recomendaciones y programas oficiales en materia educativa, lo intercultural sigue siendo un elemento retórico presente en los discursos educativos, pero que no siempre afecta a las prácticas y a la investigación. Por ello, es relevante desarrollar iniciativas de educación superior orientadas a adquirir conocimientos, destrezas y actitudes que nos permitan interactuar adecuadamente en una sociedad que se reconoce pluricultural y defiende ideales de justicia social y participación democrática.

\section{Epistemología desde un enfoque intercultural}

El paradigma intercultural se propone como metáfora de la diversidad (Aguado, 2009), como mirada que contempla y permite pensar la diversidad como condición humana. Esta aceptación implica renunciar a cualquier epistemología que pretendiera convertirse en vía única para construir conocimiento: "la diversidad del mundo es inagotable y esa diversidad todavía carece de una adecuada epistemología. En otras palabras, la diversidad epistemológica del mundo todavía está por construir" (Santos, 2009: 51).

El contexto político es frecuentemente silenciado en la reflexión y debate sobre cómo se genera el conocimiento. Sin embargo, como señala Bourdieu, "los conflictos epistemológicos son siempre, inseparablemente, conflictos políticos" (2008: 15). La investigación se promueve en el seno de cada estado bajo las directrices establecidas desde las políticas públicas de ciencia y tecnología. Los gobiernos, con sus ideologías e intereses específicos, articulan la agenda de investigación e innovación donde se delimitan prioridades temáticas, modos de producción científica, resultados esperados e impactos. Como imagen basta pensar que el plan estatal de Investigación Científica, Técnica y de Innovación fue establecido en nuestro país en el período 2013-16 por el Ministerio de Economía y Competitividad, lo que explica ya bastante de la orientación que tiene este plan. El desarrollo científico no es ajeno a los intereses neoliberales $\mathrm{y}$, en consecuencia, la búsqueda de la igualdad de oportunidades sociales no constituye el motor de cambio que mueva a la ciencia.

La epistemología en los estudios interculturales se configura como respuesta a cuestiones éticas que plantean el sentido de la propia investigación: con qué mirada del mundo, para qué investigar, con quiénes, cómo.
En los apartados que siguen vamos a compartir algunas ideas a las que hemos llegado en nuestra reflexión y experiencia de trabajo.

No se trata de respuestas únicas ni respuestas concluyentes sobre estos interrogantes. Desde un enfoque intercultural retomamos elementos interpretativos y sociocríticos para generar alternativas al pensamiento dominante que impone una lógica única, dejando abierta la posibilidad de incorporar otras lógicas que contribuyan al desarrollo de una ciencia orientada a lograr una sociedad más dialógica.

\section{Complejidad}

La comprensión no tiene fin y por lo tanto no puede producir resultados definitivos; es el modo específicamente humano de vivir, ya que cada persona necesita reconciliarse con el mundo en que ha nacido como extranjero y en cuyo seno permanece siempre extraño a causa de su irreducible unicidad (Arendt, 1995: 30).

Leyes generales, categorías definidas, patrones fijos de pensamiento proveen agilidad en la forma de entender y convivir en el mundo. Nos procura seguridad vivir y aceptar un sistema establecido en el que conformamos la experiencia gratificante de manejar certezas como garantía de conocimiento y como aval de nuestras decisiones. Olvidarnos del oficio de pensar nos traslada a un universo manejable en que podemos relajar la extenuación de la reflexión constante.

Estos pensamientos congelados, parece decir Sócrates, son tan cómodos que podemos valernos de ellos mientras dormimos; pero si el viento del pensamiento, que ahora soplaré con vosotros, os saca del sueño y os deja totalmente despiertos y vivos, entonces os daréis cuenta de que nada os queda en las manos sino perplejidades, y que lo máximo que podéis hacer es compartirlas unos con los otros (Arendt, 1995: 125).

Buena parte de la construcción de la ciencia ha venido privilegiando una razón fiel a la idea de estabilidad y orden. El fin de explicar y predecir, incluso el fin de explorar -aparentemente más abierto y dinámico-, ha operado desde esquemas rígidos que ofrecen una simplificación excesiva de las situaciones y hechos investigados. La realidad es 
analizada a partir de su fragmentación, obviando la interconexión necesaria de todo lo que vive.

Por ello, un conocimiento científico que no resulte ajeno a la experiencia de vida exige buscar alternativas de investigación que recuperen la complejidad de nuestros escenarios y de las relaciones que albergan. Esa complejidad asombrosa que lleva a un conocimiento en espiral, sin término, sin cesar de realizar círculos concéntricos (Morin, 2004).

Lo complejo, como indica Rauber (2014), alude en primera instancia a la multiplicidad de factores intervinientes en un fenómeno. Y a partir de ello, lo que completa el significado de la complejidad, es la variabilidad con la que unos factores intervienen sobre otros en función de los contextos dinámicos que se dan a lo largo del tiempo. En consecuencia, debemos rechazar la idea de lo absoluto, lo incuestionable, la permanencia.

Lo complejo no puede ser una palabra que sirva para explicar algo. Al contrario, es algo que debe ser explicado.

En este sentido se hace necesario interpelar a una "hermenéutica de la sospecha" (Rauber, 2006: 157). Sospechar del orden, de lo establecido, de lo resuelto; abrirnos hacia nuevas búsquedas y significados que hagan que los criterios con los cuales hemos creído entender el mundo resulten ahora resbaladizos entre nuestras propias manos. Implica una búsqueda de nuevas categorizaciones, distintas a las propuestas comúnmente por las disciplinas sociales: "Mejores palabras para comprender y nombrar [...] el poco o mucho saber que la experiencia de la relación con el otro, distinto de mí y sobre todo distinto de este fantasma que aúna al 'nosotros los normales', puede darme. Este fantasma que no es más que la negación del otro en mî́" (Pérez de Lara, 2006: 187).

\section{Conocimiento en relación}

No hay más objetividad posible que esa: una lo más honesta posible declaración de la propia subjetividad (Lizcano, 2006: 126).

Una epistemología acorde con la mirada intercultural supone romper con toda aspiración de neutralidad u objetividad (Ballesteros y Mata, 2014). El investigador social está inmerso en la realidad en la que se producen las situaciones que investiga, formando parte de ella. En consecuencia no aspira a construir conocimiento sobre la sociedad como algo ajeno que no le pertenezca. En su lugar, la investigación queda comprometida con la búsqueda de sentidos.

La materia prima con la que trabaja el investigador cualitativo está formada por interpretaciones particulares de la experiencia vivida. No hay, por tanto, un "objeto" de la investigación que podamos describir y explicar sino un conjunto de subjetividades, incluida la propia del investigador: distintas interpretaciones a partir de las cuales confiamos en que sea posible alcanzar un significado intersubjetivo y por tanto un cierto conocimiento común, si bien situado y siempre revocable (Ballesteros y Mata, 2006: 22).

Este conocimiento que se genera es una producción constructiva e interpretativa. El construccionismo rompe con la dicotomía sujeto-objeto, puesto que ninguna de ellas tiene una existencia al margen de la otra (Iñiguez, 2003), rechazando cualquier forma de esencialismo, cualquier forma de estabilidad. Construir conocimiento desde el enfoque intercultural implica relación y escucha, apertura a otros posibles, como indica Piussi:

Esta competencia de estar ahí [...] atesora la pasividad, un reposar la mente y la acción y detenerse también dentro de sí, en su propia sombra, para escucharse, abrirse dentro, mientras se escucha al otro: una pasividad eficaz, un darse y dar el tiempo necesario para hacer y hacerse preguntas, para escuchar y acoger también las que no tienen respuesta (Piussi, 2006: 41-42).

La intersubjetividad alude expresamente a lo intercultural como proceso comunicativo, proceso en el que los actores asumen la reciprocidad de las perspectivas. El conocimiento nace en el espacio de la interacción social, donde busca y comparte sentidos; un conocimiento orientado por otros valores vinculados a una ética del conocimiento que propone la equidad y justicia como fin y proceso de investigación. Desde esta perspectiva, la investigación no consiste en la búsqueda de una verdad como única, sino en el diálogo de saberes sobre los problemas sociales que demandan una mirada renovada para lograr otras respuestas entretejidas con la propia voz de los protagonistas. 
La investigación aspira a convertirse en cuestión pública, siendo pública la realidad que construimos, las problemáticas que identificamos, su estudio y las acciones que se derivan.

\section{Conocimiento para la emancipación}

Me gusta ser persona porque la Historia en que me hago con los otros y de cuya hechura participo es un tiempo de posibilidades y no de determinismo (Feire, 1997:52)

El desarrollo de la ciencia ha ido valorando un saber que se legitima más por reunir ciertas características que por su contribución a interpretaciones o respuestas ante cuestiones sociales. Avanzar hacia un conocimiento que promueva equidad y justicia no ocupa una prioridad entre las agendas de investigación oficiales que pretenden ser el canon que oriente la actividad investigadora reconocida.

En términos de valoración y acreditación científica se destaca más una investigación que reúna requisitos como el control, exactitud de medida, precisión, suficiencia y representatividad de muestras, capacidad de generalización, entre otros, aunque el problema que estudie tenga una naturaleza banal. Estas investigaciones cuentan con más reconocimiento y apoyo que otras orientadas a lograr respuestas intersubjetivas y fragmentadas a cuestiones derivadas de la desigualdad social.

Preguntarnos para qué y para quién investigamos nos lleva de la mano a tomar posición en la relación entre ética y ciencia, entre conocimiento y vida (Ghiso, s.f.).

La ciencia, como si de un doble espejo se tratara, ha ido forjando un muro entre las realidades investigadas y los propios investigadores, bajo la extraña creencia -tal vez-de que con este distanciamiento conseguía aproximarse a una forma de objetividad supuestamente existente y necesaria. Buena parte de la tarea científica, indica Lizcano (2006), omite o niega la posibilidad de creación de realidad social, limitándose a presentar al agente como paciente, consolidando así el mito de la ciencia. En palabras de Santos es lo que llama pensamiento abismal, cuyo interés empieza por negar la posibilidad de llegar a un conocimiento desde la participación de todos los implicados:

Como un producto del pensamiento abismal, el conocimiento científico no es socialmente distribuido de un modo equitativo, no podría serlo, fue diseñado originariamente para convertir este lado de la línea en un sujeto de conocimiento, y el otro lado en un objeto de conocimiento (Santos, 2009: 56).

Urge así construir el conocimiento desde una perspectiva que ponga en valor la necesidad de trabajar por la justicia y equidad social, un conocimiento que humanice nuestras relaciones en el mundo. Un conocimiento que "apele a nuestra responsabilidad de actuar racionalmente $[\ldots]$ y con fuerza en busca de un sistema histórico mejor" (Wallerstein, 2002:8).

En este sentido Santos (2009) subraya la necesidad de una ecología de saberes que ponga en valor el conocimiento como práctica de intervención en la vida social:

La ecología de saberes no concibe los conocimientos en abstracción; los concibe como prácticas de saberes que permiten o impiden ciertas intervenciones en el mundo real. Una pragmática epistemológica está sobre todo justificada porque las experiencias vitales de los oprimidos son primariamente hechas inteligibles para ellos como una epistemología de las consecuencias. En su mundo vital, las consecuencias son primero, las causas después.

La ecología de saberes está basada en la idea pragmática de que es necesario revalorizar las intervenciones concretas en la sociedad y en la naturaleza que los diferentes conocimientos pueden ofrecer (Santos, 2009: 59).

La ecología de saberes rescata y valora los saberes populares como forma de avance de la propia ciencia, evitando la separación sujeto-objeto, favoreciendo formas de conocimiento y acción que emergen del imaginario social (Lizcano, 2006). El saber popular entra en diálogo con otro tipo de saberes; investigadores e investigados pueden cooperar en la construcción del conocimiento desde la lógica de la relación simétrica y horizontal (Vasilachis de Gialdino, 2009).

Al tiempo, la ecología de saberes desdibuja la frontera entre el saber y la acción desde una perspectiva emancipadora. Emanciparse implica la posibilidad de construir la propia identidad social 
de forma autónoma, más allá de las adscripciones sociales establecidas, más allá de las expectativas y trayectorias a seguir trazadas desde las estructuras de poder que marcan las relaciones de dominación y sometimiento. En consecuencia, emanciparse implica necesariamente el reconocimiento de la agencialidad de las personas: la confianza en que las personas puedan apropiarse del carácter público de la vida social como espacio que nos pertenece, donde proyectar y construir colectivamente la vida social.

\section{Nuestra trayectoria como grupo de investigación: Aportaciones, dilemas y retos}

Nuestras líneas de investigación y docencia las venimos desarrollando dentro del Grupo INTER (www.uned.es/grupointer), integrado por profesoras y estudiantes de diversos departamentos e instituciones educativas y de investigación de España y de otros países, como espacio para la reflexión y la investigación sobre diversidad cultural y educación. Iniciado en 1995, lo hemos ido consolidando a lo largo de los últimos años por medio de proyectos de investigación subvencionados por organismos oficiales. A raíz de nuestra participación como coordinadoras en el Proyecto INTER (Programa Sócrates, Comisión Europea, 2003-2005) el grupo se ha ampliado con personas pertenecientes a distintas instituciones españolas y europeas, y tras la financiación por parte de la Agencia Española de Cooperación Internacional (AECI) de tres proyectos bilaterales de Cooperación Internacional (PCI) y la coordinación del proyecto INTER-ALFA (Programa ALFA de Cooperación Académica entre la Unión Europea y América Latina. Comisión Europea, 2007-2009), el grupo se ha enriquecido con la incorporación de instituciones latinoamericanas, como la Universidad Veracruzana y la Universidad de Tarapacá, entre otras.

Nuestro punto de partida, en consonancia con el enfoque intercultural descrito en el primer apartado de este artículo, es colocar la diversidad cultural como foco de toda reflexión pedagógica, considerando que tal diversidad, lejos de ser una excepción, es la norma en toda situación educativa. Nuestras actividades se han dirigido a revisar conceptos clave en el ámbito educativo, como son los de cultura(s), diversidad, igualdad de oportunidades, calidad y equidad, justicia social, racismo, mediación, ciudadanía y participación. Al mismo tiempo, a conceptualizar y desarrollar el enfoque intercultural tanto en sus presupuestos teóricos como en sus implicaciones prácticas.

En docencia nos proponemos sensibilizar en torno a estas cuestiones y plantear vías de actuación educativa en contextos formales (escolaridad obligatoria) y no formales (educación social, voluntariado, mediación social, aprendizaje activo de la ciudadanía).

Desde el curso 2011/12 el grupo INTER imparte un Máster Oficial adaptado al EEES, el Máster Eurolatinoamericano en Educación Intercultural con el objeto de formar profesionales e investigadores que adopten una mirada intercultural.

Nuestro trabajo siempre va acompañado de dilemas que ponen el foco en la relación entre ética e investigación y tienen que ver con la reflexión y práctica desde un enfoque intercultural. El debate no nos lleva a respuestas concluyentes ni puntos de vista compartidos por todos los miembros del grupo. Siguen siendo temas que abordamos en nuestros encuentros y en las reflexiones que compartimos, como las que siguen.

\section{El dilema de la categorización, ¿cómo abordamos el tema de las "categorías" en nuestra praxis?}

Los seres humanos necesitamos la categorización, pero esta se hace patológica cuando se entiende que la categoría es definitiva, impidiendo así que los individuos consideren las borrosas fronteras de la misma, y no digamos que puedan revisar sus categorías. Taleb, 2008: 57-58

Dando un nombre a algo nos hemos limitado a ponerlo en una categoría, y pensamos que lo hemos comprendido; no lo miramos más atentamente. Pero si no lo nombramos estamos obligados a mirarlo. O sea, abordamos la flor, o lo que fuere, con un sentido de novedad, con una calidad nueva de examen: la miramos como si nunca la hubiésemos mirado antes. Krishnamurti, 1998:405

El cerebro humano necesita organizar de alguna forma toda la información que le llega del exterior. La única forma es hacerlo mediante categorías y esquemas mentales, que nos ayudan a registrar y procesar los datos, pero estas categorías no son 
innatas, no tenemos un repertorio preestablecido al nacer, se van conformando según vamos adquiriendo nuevos aprendizajes. Puesto que el aprendizaje está condicionado por el contexto social en el que se desarrolla el ser humano, estas categorías y esquemas mentales están condicionados socialmente, y se van conformando en función de nuestra relación con otras personas.

Tenemos una excelente capacidad para categorizar el mundo que nos rodea, para reducir la complejidad de nuestro entorno, ofreciéndole un orden, permitiendo hacerlo más predecible y mejorable (Bruner, 1971; citado en Téllez, 2005). Este es precisamente el mecanismo por el que se generan los estereotipos y los prejuicios. Como afirma Jiménez Frías (2005), tanto el estereotipo como el prejuicio se relacionan con los procesos de categorización del pensamiento, con la capacidad de utilizar categorías para ordenar y simplificar la percepción de la realidad.

Los estereotipos son estructuras cognitivas que contienen los conocimientos, creencias y expectativas del observador acerca de algún grupo humano. Creencias generalizadas y generalmente erróneas sobre atributos y conductas que caracterizan a los miembros de un grupo (Allport, 1954; citado en Jiménez, 2005). Las expectativas respecto de una persona, que surgen tras clasificarla en una determinada categoría o un grupo específico, tienen claras consecuencias en las decisiones que se toman respecto de opciones educativas, itinerarios formativos, expectativas de éxito o fracaso, etc.

A pesar de las consecuencias negativas de clasificar y utilizar categorías ¿por qué seguimos utilizándolas? Hemos preguntado a algunas compañeras del grupo, a quienes agradecemos que hayan compartido sus ideas, ya que ayudan a ejemplificar cómo afrontamos los dilemas que se nos plantean.

[...] yo creo que nuestras investigaciones no pueden estar exentas de categorías porque yo, sin categorías, no puedo ordenar ideas o pensar. Otra cosa es que sea consciente de que lo estoy haciendo y de que intente no dejar que esas categorías dirijan mi investigación (si no quiero hacerlo). No se trata tanto de huir de categorías (desde que nacemos categorizamos sin parar para intentar comprender nuestro mundo), sino ser críticas con ellas e intentar analizar los mecanismos que subyacen a estas categorías y todo lo que pueden provocar (Carmen Osuna, Grupo INTER).

Para mí ningún pensamiento está exento de categorías porque para pensar necesitamos categorizar, es decir, agrupar ideas para hacerlas más abstractas y poder predecir en cierta medida. Cuando investigo, voy con mis categorías, pero con la intención de cambiarlas descubriendo cuáles son las que emplea la gente en el contexto en el que estoy trabajando. [...] Cuando consigo descubrir algunas de las categorías que usa la gente en el contexto que investigo las uso de dos formas: primero para aprender a preguntar de manera significativa y segundo para complejizar y a veces descartar las categorías con las que yo he empezado el trabajo. Además de esto tengo más o menos clara otra idea: que para conocer a la gente de persona en persona las categorías sirven de muy poco, porque nos empujan a asumir en vez de preguntar (Margarita del Olmo, Grupo INTER).

Lo esencial es no presuponer comportamientos determinados, ni crear expectativas en función del grupo al que se autoadscriben las personas, sino dejar que cada uno se describa y se defina como quiera, o no se adscriba a ningún grupo previamente definido.

Lo más grave de clasificar a las personas son las consecuencias que se derivan de dicha clasificación cuando no han sido elegidas voluntariamente. No obstante, reconocemos el interés que pueda tener en determinadas circunstancias autoadscribirse a una categoría determinada o varias, ya sea por opción ideológica, política, económica.

En las escuelas de Nueva York, por ejemplo, categorizan a los estudiantes según la autoadscripción que hacen ellos mismos. Una de las alumnas me dijo que ella se considera "Brown", pero siempre pone que es 'afroamericana' porque es lo que 'se supone que es'. Según ella, en las escuelas se hace esto porque a partir de un cierto número de alumnos "diferentes" se consiguen muchos recursos. Por una parte, creo que la parte de obtención de recursos tiene que ver con asumir y 
consolidar la desigualdad. Por otra parte, en una exposición leí un artículo de los años 70, en el que se relataba cómo los estudiantes negros pedían el fin del enfoque "color blind" y hablaban de la necesidad del "color-consciousness". Lo relacioné con el tema de los indígenas en México, pensando que debe haber razones de mucho peso para que la gente quiera ser étnicamente identificada y clasificada, y creo que quizá no todo tenga que ver con posibles ventajas. Tampoco creo que la solución sea ignorarlas, están ahí y eso no se puede obviar, pero sí cuestionar una y otra vez (Patricia Mata, Grupo INTER).

La categorización no está exenta de riesgos y en muchas ocasiones se están enmascarando situaciones de poder, que buscan mantener las desigualdades sociales. Como afirma Aguado "la prioridad debería ser garantizar una distribución de recursos materiales y humanos que hiciera posible experiencias educativas enriquecedoras para todos" (Aguado, 2009:21).

\section{El dilema sobre el relativismo, ¿es posible el relativismo en la investigación aplicada?}

Abrir la mirada y tratar de comprender desde la posición de quienes protagonizan una situación social ha sido el objetivo planteado desde el enfoque interpretativo aplicado en nuestro caso a los estudios interculturales.

Abrir la mirada supone así, desde esta perspectiva, el intento de abandonar en nuestro trabajo de campo el juicio previo que tenemos sobre el tema que investigamos para comprender las causas y mecanismos por los que se producen los hechos y se legitiman en un grupo social determinado (Monge, 2009). Al tiempo supone un compromiso ético del investigador en relación con el tema de estudio: la comprensión del significado de las prácticas sociales en un grupo determinado solo pasa por entender las razones de su dinámica. Se trata así de buscar otras perspectivas de sentido común y eso solo puede ser posible en la medida en que utilicemos el relativismo cultural como herramienta de trabajo.

Ahora bien, cuando nuestra investigación pretende incidir en la práctica, cuando nos planteamos una acción para mejorar la vida de los grupos, ¿hasta qué punto podemos hacer compatible acción y relativismo?, ¿deberíamos ser únicamente observadores de la acción?, ¿no podríamos emitir un juicio en tanto miembro del grupo?

Son dilemas no resueltos, donde vemos posturas diversas y cambiantes según avanzamos en nuestra trayectoria, como mostramos en estas opiniones recabadas entre algunas de nosotras:

La acción de una investigación concebida desde el relativismo cultural tiene que estar orientada a facilitar la acción que las personas involucradas en la investigación deseen hacer y no desde lo que yo piense que se podría o debería realizar, incluyendo la posibilidad de no realizar ninguna acción si las personas involucradas así lo prefieren (Margarita del Olmo, Grupo INTER).

En otra reflexión recogida entre nosotras vincula acción con investigación acción participativa (IAP) como vía posible de conciliar acción y relativismo entendido desde una dimensión metodológica que no anulara la capacidad de juicio ante la injusticia y desigualdad:

La idea de una "investigación orientada a la acción" dice poco sobre qué tipo de investigación y qué tipo de acción. Tiene más sentido si añadimos la idea de participación. Entonces la investigación y la acción se convierten en colectivas y el foco se pone en el proceso. Así podrían generarse procesos en los que se incorporen puntos de vista diferentes y sería posible llegar a consensos y propuestas creativas con capacidad para cambiar las condiciones previas (Patricia Mata, Grupo INTER).

No obstante, la conciliación de IAP y relativismo sigue mostrando otras tensiones que nos resitúa de nuevo ante otros problemas éticos:

Creo que en estas investigaciones se tiende a perder de vista las inevitables relaciones de poder que siempre existen en cualquier relación humana y que pueden ser limadas pero que finalmente harán presencia [...]. Podemos poner todos nuestros sentidos en el relativismo cultural para intentar 
comprender, desde otro punto de vista, qué está pasando o qué prioridades o valores prevalecen en uno u otro contexto... Pero por mucho que nuestras investigaciones intenten huir de ellas, siempre habrá relaciones de poder y los inevitables dilemas éticos (Carmen Osuna, Grupo INTER).

Nuestras preocupaciones forman parte del propio enfoque intercultural, asumiendo que es complejo y que no ofrece soluciones simples (Mata y Ávila, 2010). Nuestro reto es seguir avanzando en investigación con nuestros dilemas e incertidumbres, incorporándolos como parte de la investigación misma. Orientar la investigación como práctica intercultural y desarrollarla mediante metodologías horizontales que favorezcan la intersección entre sujetos y, por tanto, por la intersección de saberes (Pérez Daniel, 2012:11). Como intersección, el encuentro entre sujetos y saberes implica diálogo.

\section{Conclusiones}

Nuestra propuesta de una epistemología intercultural se orienta desde una mirada interpretativa y participativa, definida por tres principios que caracaterizan una forma de conocimiento: interés por la complejidad de las situaciones vitales; relación dialógica como expresión de la intersubjetividad; búsqueda de la justicia y equidad a través de la emancipación social.

Nuestra trayectoria como Grupo Inter, tanto investigadora como de docencia, no es ajena a las dificultades y vicisitudes que plantea este enfoque.

Aunque reconocemos nuestras contradicciones y distancias entre decires y haceres, planteamos esta epistemologia como un reto, un horizonte al que aproximarnos. En el camino, los errores también nos enseñan.

Identificarlos y pensarlos es nuestro aprendiazaje como investigadoras comprometidos con una ética del trabajo.

\section{Referencias Citadas}

Abdallah-Pretceille, $\mathrm{M}$.

2006 El paradigma intercultural como mirada hacia la diversidad. Actas Congreso INTER. Madrid: Servicio de Publicaciones UNED. www.uned.es/congreso-intereducacion-intercultural (5 abril 2015).

Aguado, T.

2009 El enfoque intercultural como metáfora de la diversidad en educación. En T. Aguado y Del Olmo Margarita (coords.) Educación Intercultural. Perspectivas y propuestas, 15-28. Madrid: Ramón Areces.

Aguado, T. (coord.)

2010 Diversidad Cultural y Logros de los Estudiantes en Educación Obligatoria. Lo que Sucede en las Escuelas. Madrid: MEC.

Asociación de Enseñantes con Gitanos

1996 LaAsociación de Enseñantes con Gitanos y la Educación Intercultural. Boletín de Centro de Documentación- AECG, $\mathrm{N}^{\circ} 12$.

Ballesteros, B.; Mata, P.

2014 Sentido y forma de la investigación cualitativa. En B. Ballesteros (coord.) Taller de Investigación Cualitativa (pp. 12-46). Madrid: UNED.

Freire $\mathrm{P}$.

1997 Pedagogía de la Autonomía. México: Siglo XXI.

Ghiso, A.

s.f. Entre el Hacer lo que se Sabe y el Saber lo que se Hace. Una Revisión sui Géneris de las Bases Epistemológicas y de las Estrategias Metodológicas. http://www.alboan.org/ archivos/538.pdf (10 abril 2015)

Iñiguez, L.

2003 La psicología social en la encrucijada postconstruccionista. Historicidad, subjetividad, performatividad, acción.
Conferencia de apertura del XII Encontro Nacional da ABRAPSO. Estratégias de invenção-a Psicologia Social no contemporáneo 15, 16 e 17. Porto Alegre: Pontifícia Universidade Católica do Rio Grande do Sul PUCRS.

Jiménez Frías, R.

2005 Estereotipo y Prejuicio. En B. Malik y M. Herraz (Coords.) Mediación Intercultural en Contextos Socioeducativos, pp. 47-66. Málaga: Aljibe.

Fundación Krishnamurti Latinoamericana

1998 Obras Completas J. Krishnamurti. Años 1933-1967. Percepción sin Opciones. Traducido por A. Clavier. Tomo V (1948-1949). Buenos Aires: Editorial KIER.

Lizcano, E.

2006 Metáforas que nos Piensan. Sobre Ciencia, Democracias y otras Poderosas Ficciones. Madrid: Traficantes de sueños.

Mata, P.; Ávila, A.

2010 Informe global de análisis de necesidades. En B. Malik, L. Lobo, V. Espinosa y A. Ávila (coords.) Un Máster en Educación Intercultural para Europa y América Latina: Necesidades, Currículo e Implantación, pp. 55-69. Madrid: INTER-ALFA

Mañeru Méndez, A.

2006 Sosiego y placer en la educación. En A. M. Piussi y A. Mañeru Méndez (coords.) Educación, Nombre Común Femenino (pp. 66-76). Barcelona: Octaedro.

Monge, F.

2009 A vueltas con el relativismo. En T. Aguado y M. del Olmo (eds.) Educación Intercultural. Perspectivas y Propuestas, pp. 103-116. Madrid: Proyecto ALFA.

Morin, E.

2004 Epistemología de la complejidad. Gaceta de Antropología, 20, artículo 02. http://hdl.handle.net/10481/7253 (8 abril 2015). 
Olivé, L.; Santos, B.; Salazar de la Torre, C.; Antezana, L.H.; Navia Romero, W.; Tapia, L.; Valencia García, G.; Puchet Anyul, M.; Gil, M.; Aguiluz Ibargüen, M. y Suárez, H. J. 2009 Pluralismo Epistemológico. CLACSO Coediciones. La Paz: CLACSO - Muela del Diablo Editores - Comunas - CIDES - UMSA.

Pérez Daniel, M. R.

2012 Prólogo. En M.R. Pérez Daniel y S. Sartorello (coords.) Horizontalidad, Diálogo y Reciprocidad en los Métodos de Investigación Social y Cultural, pp. 11-14. México: CENEJUS

Pérez de Lara Ferré, N.

2006 El otro (y la otra; lo otro). En A. M. Piussi y a. Mañeru Méndez (coords.) Educación, Nombre Común Femenino, pp. 66-76. Barcelona: Octaedro.

Piussi, A. M.

2006 El sentido libre de la diferencia sexual en la educación. En A. M. Piussi y A. Mañeru Méndez (coords.) Educación, Nombre Común Femenino, pp. 15-45. Barcelona: Octaedro.

Rauber, I.

2006 América Latina. Sujetos políticos. Rumbos Estratégicos y Tareas Actuales de los Movimientos Sociales y Políticos. Santo Domingo: Pasado y Presente XXI.

Rauber, I.

2014 Gobernar las coyunturas. Elementos Teórico-metodológicos para el Análisis de las Coyunturas Políticas. (15 marzo 2014) http://isabelrauber.blogspot.com.es/2014/03/gobernar-lascoyunturas.html (2 abril 2014).

Rey von Allmen, $M$.

2010 The Intercultural Perspective and its Development Through Cooperation With the Council of Europe, in C.A. Grant \& A. Portera (eds.) Intercultural and Multicultural Education. Enhancing Global Interconnectedness, pp. 33-48. New York: Routledge.

Taleb, N.

2008 El Cisne Negro: El Impacto de lo Altamente Improbable. Madrid: Paidós.

Téllez, J. A.

2005 Mentes en Relación. En B. Malik y M. Herraz (Coords.) Mediación Intercultural en Contextos Socio-educativos, pp. 32-45. Málaga: Aljibe.

Téllez, J. A.; Mata, P. Sutil, I. Malik, B.

2005 Orientación Intercultural. Madrid: Sanz y Torres.

Vasilachis de Giladino, I.

2009 Los fundamentos ontológicos y epistemológicos de la investigación cualitativa. Forum: Qualitative Social Research. Volumen 10, No. 2, Art. 30. http://www.qualitative-research. net/index.php/fqs/article/view/1299/2778\#g7 (15 abril 2015).

Wallerstein, I.

2002 Conocer el Mundo, Saber el Mundo: el fin de lo Aprendido, una Ciencia Social para el siglo XXI. México: Siglo XXI. 
